\title{
A Smooth Interface Method for Simulating Liquid Crystal Colloid Dispersions
}

\author{
Ryoichi Yamamoto§, Yasuya Nakayama, and Kang Kim \\ Department of Physics, Kyoto University, Kyoto 606-8502, Japan and PRESTO, \\ Japan Science and Technology Agency, 4-1-8 Honcho, Kawaguchi 332-0012, Japan.
}

\begin{abstract}
A new method is presented for mesoscopic simulations of particle dispersions in liquid crystal solvents. It allows efficient first-principle simulations of the dispersions involving many particles with many-body interactions mediated by the solvents. Demonstrations have been performed for the aggregation of colloid dispersions in two-dimensional nematic and smectic- $\mathrm{C}^{*}$ solvents neglecting hydrodynamic effects, which will be taken into account in the near future.
\end{abstract}

Submitted to: J. Phys.: Condens. Matter

\section{Introduction}

Dispersions of small particles in host fluids such as colloid suspensions and emulsions are of considerable technological importance and appear often in our everyday life as paints, foods, and drugs for example. Many kinds of exotic interactions were found between particles mediated by the host fluids including screened Coulombic [1], depletion [1, fluctuation induced [2], and surface induced [3] forces. A striking example occurs when spherical particles are immersed in a liquid-crystal (LC) solvent in the nematic phase 4. For a single particle, the orientation of the solvent molecules is distorted due to the anchoring of the solvent molecules at the particle surface. Extensive studies have been done on this effect, and several characteristic configurations of the nematic field around a spherical particle have been identified [5, 6, 7, 8, 9, 10. When the strength of anchoring is increased so that normal anchoring is preferred, the solvent changes from quadrupolar to dipolar symmetries around the particle. When multiple particles are immersed in the solvent, long-range anisotropic interactions are induced between particles due to elastic deformations of the nematic field [11, 12, 13, 14. The anisotropic interactions can have a pronounced effect not only on the local correlations of the particles [1], but also on their phase behavior [15, 16, 17, 18, 19, 20] and on their mechanical properties 16. It has been reported also that a similar, but somewhat different situation occurs when colloid particles are immersed in smectic- $\mathrm{C}^{*}$ films [21, 22, 23]. The purpose of $\S$ To whom correspondence should be addressed (ryoichi@scphys.kyoto-u.ac.jp) 
our project is to develop an efficient method suitable for simulating colloid dispersions immersed in LC solvents by using smooth interface between colloids and solvents. Effects of hydrodynamics is omitted in the present paper though it is very important [24, 25]. That will be taken into account in the near future also through the smooth interface 26]. The same method is applicable for charged colloid dispersions where the charge density on colloid surface is given by a smooth function [27].

\section{Mesoscopic Model}

Since analytical approaches for investigating complex materials such as LC colloid dispersions considered here are extremely difficult, computer simulations are the most promising tool to investigate their static and dynamical properties. In colloid dispersions, the host fluid molecules are much smaller and move much faster than the dispersed particles. This enables us to use coarse grained mesoscopic variables, which should be described by the hydrodynamics, for the host fluids rather than treating them as fully microscopic objects [28, 29. In the case of charged colloid suspensions for example, a mesoscopic method for the first principle simulations can be derived by treating the counter ions as a charge density [30]. For the particle dispersions in LC solvents considered here, the mesoscopic free energy $\mathcal{F}$ of the system can be given by functionals of the director $\mathbf{n}(\mathbf{r})$, a common direction on which solvent molecules are aligned on average with a constraint $|\mathbf{n}(\mathbf{r})|=1$, for a given particle configuration $\left\{\mathbf{R}_{1} \cdots \mathbf{R}_{N}\right\}$ :

$$
\mathcal{F}\left(\mathbf{n}(\mathbf{r}) ;\left\{\mathbf{R}_{1} \cdots \mathbf{R}_{N}\right\}\right)=\mathcal{F}_{e l}+\mathcal{F}_{s}=\int d \mathbf{r} f_{e l}(\mathbf{r})+\oint d S f_{s}(S),
$$

where

$$
f_{e l}(\mathbf{r})=\frac{1}{2}\left[K_{1}(\nabla \cdot \mathbf{n})^{2}+K_{2}(\mathbf{n} \cdot \nabla \times \mathbf{n})^{2}+K_{3}\{\mathbf{n} \times(\nabla \times \mathbf{n})\}^{2}\right]
$$

is the Frank free energy 31] which presents the elastic energy density of the nematic solvent at $\mathbf{r}$ and the surface free energy

$$
f_{s}(S)=\frac{W}{2}\left[1-(\mathbf{n} \cdot \boldsymbol{\nu})^{2}\right]
$$

controls anchoring of the LC solvent at the surface element $S$ of the particle. The coefficients $K_{1}, K_{2}$, and $K_{3}$ are the splay, twist, and bend elastic constants, respectively, and the single constant approximation $\left(K=K_{1}=K_{2}=K_{3}\right)$ reduces Eq.(2) to the simpler form

$$
f_{e l}(\mathbf{r})=\frac{K}{2}\left[(\nabla \cdot \mathbf{n})^{2}+(\nabla \times \mathbf{n})^{2}\right] .
$$

$W$ is the surface anchoring constant, and $\boldsymbol{\nu}$ is the unit vector normal to the colloid surface [7, 8]. The saddle-splay elastic term [8] is not considered here. The integral in the first term on the right hand side of Eq.(11) runs over the whole solvent volume excluding the particles, and that in the second term runs over all solvent-particle interfaces. A simple scaling argument tells us $\mathcal{F}_{e l} \propto K a^{d-2}$ and $\mathcal{F}_{s} \propto W a^{d-1}$ with $a$ and $d$ being the particle 
radius and the system dimension, respectively, thus the state of the dispersion should be controlled by the ratio $\mathcal{F}_{s} / \mathcal{F}_{e l} \propto W a / K$. Although this type of free energy functional is sufficient for single particle problems, it is not useful for simulating colloid dispersions involving many particles using molecular dynamics (MD) or Brownian type methods because the coupling between solvent and the particles is given implicitly by limiting the integration space in both $\mathcal{F}_{e l}$ and $\mathcal{F}_{s}$. This produces mathematical singularities at the interface when one calculates the force, $\mathbf{f}_{n}^{P S}=-\partial \mathcal{F} / \partial \mathbf{R}_{n}$, acting on each particle mediated by the LC solvents. Calculation of the force is crucial for performing efficient simulations of many particle systems. Another serious problem of this type of functional is that in order to give correct boundary conditions at the particle-solvent interface, one has to use appropriate coordinates for performing grid-based numerical simulations rather than the usual Cartesian coordinates. This is generally difficult for particles with non-spherical shapes or for systems involving many particles even when each particle has a spherical shape. Also this makes the use of the periodic boundary condition difficult, which is a fatal situation for simulating bulk materials.

To overcome these problems, we have modified Eqs.(11), (3), and (41) by using a smooth interface between the solvent and the particles so that the coupling is given explicitly in the integrand through the interface [32]. The new free energy functional we propose has the form

$$
\begin{aligned}
\mathcal{F}\left(\mathbf{n}(\mathbf{r}) ;\left\{\mathbf{R}_{1}\right.\right. & \left.\left.\cdots \mathbf{R}_{N}\right\}\right) \\
& =\int d \mathbf{r}\left[1-\sum_{i=1}^{N} \phi_{i}(\mathbf{r})\right] f^{e l}(\mathbf{r})+\int d \mathbf{r} \sum_{i=1}^{N} \xi\left(\nabla \phi_{i}\right)^{2} f_{i}^{s}(\mathbf{r})
\end{aligned}
$$

with

$$
\begin{aligned}
f^{e l}(\mathbf{r}) & =\frac{K}{4 R_{c}^{2}} \tanh \left[R_{c}^{2}\left(\nabla_{\alpha}\left(n_{\beta} n_{\gamma}\right)\right)^{2}\right] \\
f_{i}^{s}(\mathbf{r}) & =\frac{W}{2}\left[1-\left(\frac{\nabla \phi_{i}}{\left|\nabla \phi_{i}\right|} \cdot \mathbf{n}\right)^{2}\right]
\end{aligned}
$$

for nematic solvent and

$$
\begin{aligned}
& f^{e l}(\mathbf{r})=\frac{K}{2 R_{c}^{2}} \tanh \left[R_{c}^{2}\left((\nabla \cdot \mathbf{n})^{2}+(\nabla \times \mathbf{n})^{2}\right)\right] \\
& f_{i}^{s}(\mathbf{r})=W\left[1-\frac{\nabla \phi_{i}}{\left|\nabla \phi_{i}\right|} \cdot \mathbf{n}\right]
\end{aligned}
$$

for smectic- $\mathrm{C}^{*}$ solvent. The summation convention is used for $\alpha, \beta, \gamma \in x, y, z$. The explicit form of the interfacial profile $\phi_{n}$ between dispersed particles and solvents and its spatial derivatives $\nabla_{\alpha} \phi_{n}$ are given by

$$
\phi_{n}(\mathbf{r})=\frac{1}{2}\left(\tanh \frac{a-\left|\mathbf{r}-\mathbf{R}_{n}\right|}{\xi}+1\right)
$$

and

$$
\nabla_{\alpha} \phi_{n}(\mathbf{r})=-\frac{r_{\alpha}-R_{n, \alpha}}{2 \xi\left|\mathbf{r}-\mathbf{R}_{n}\right|} \cosh ^{-2} \frac{a-\left|\mathbf{r}-\mathbf{R}_{n}\right|}{\xi}
$$




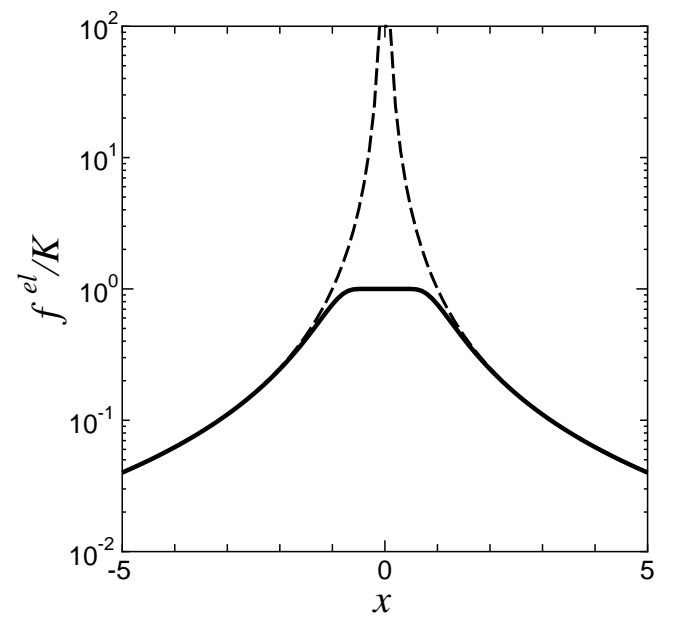

Figure 1. The elastic free energy density $f_{e l}(x)$ around a point defect at $x=0$. The dashed line is from Eq.(44), $f_{e l} \simeq K / x^{2}$, and the solid line is from Eq.(6) or (8) with $R_{c}=1, f_{e l} \simeq K \tanh \left(1 / x^{2}\right)$.

respectively with the particle radius $a$ and the interface thickness $\xi$. Note that a set of Eqs.(15)-(11) reduces to Eqs.(11), (3), and (4) for the limit $R_{c}, \xi \rightarrow 0$. A similar idea for using smooth interface was used to treat the hydrodynamic forces acting on particles dispersed in simple liquids [33], recently. For the nematic case, $f_{e l}$ is given by a function of a symmetric second-rank tensor $q_{\alpha \beta}=n_{\alpha} n_{\beta}$ rather than the director $\mathbf{n}$ itself to take into account the symmetry of the nematic director $+\mathbf{n} \leftrightarrow-\mathbf{n}$ automatically. The semi-empirical functional form $\frac{1}{R_{c}^{2}} \tanh \left[R_{c}^{2} \cdots\right]$ is applied in Eqs.(6) and (8) to avoid the mathematical divergence of the elastic free energy density at the defect centers and to limit its value to $\Delta f_{e l} \sim K / R_{c}^{2}$ and replace its core size with $R_{c}$ as shown in Figure 1 Another way to avoid the divergence would be to use the Landau-de Gennes type free energy with an order parameter $Q_{\alpha \beta}(\mathbf{r})=Q(\mathbf{r}) q_{\alpha \beta}(\mathbf{r})$, but this requires a prohibitively small lattice spacing near the defect points [34.

\section{Numerical method}

\subsection{First-principle Simulation}

The simulation procedure is as follows.

(i) For a given particle configuration $\left\{\mathbf{R}_{1} \cdots \mathbf{R}_{N}\right\}$, obtain the interface profile $\phi_{n}(\mathbf{r})$ by Eq.(101). Then we can calculate the stable (or meta-stable) nematic configuration $\mathbf{n}^{(0)}(\mathbf{r})$ which should satisfy the equilibrium condition

$$
\left.\frac{\delta \mathcal{F}}{\delta \mathbf{n}(\mathbf{r})}\right|_{\mathbf{n}(\mathbf{r})=\mathbf{n}^{(0)}(\mathbf{r})}=0
$$

under the director constraint $|\mathbf{n}(\mathbf{r})|=1$. One can perform this by numerical iterations such as the steepest descent or the conjugate gradient method. Although 
the time evolutions of $\mathbf{n}(\mathbf{r})$ should be determined by the hydrodynamic laws, we here assume $\mathbf{n}(\mathbf{r})$ follows adiabatically after $\left\{\mathbf{R}_{1} \cdots \mathbf{R}_{N}\right\}$ for the sake of simplicity.

(ii) Once $\mathbf{n}^{(0)}(\mathbf{r})$ is obtained, the force acting on each particle mediated by the nematic solvents follows directly from the Hellmann-Feynman theorem,

$$
\begin{aligned}
\mathbf{f}_{n}^{P S}\left(\left\{\mathbf{R}_{1} \cdots \mathbf{R}_{N}\right\}\right) \equiv-\frac{\partial \mathcal{F}\left(\mathbf{n}^{0}(\mathbf{r}) ;\left\{\mathbf{R}_{1} \cdots \mathbf{R}_{N}\right\}\right)}{\partial \mathbf{R}_{n}} \\
=\frac{K}{4 R_{c}^{2}} \int d \mathbf{r} \frac{\partial \phi_{n}}{\partial \mathbf{R}_{n}} \tanh \left[R_{c}^{2}\left(\nabla_{\alpha}\left(n_{\beta}^{0} n_{\gamma}^{0}\right)\right)^{2}\right] \\
\quad+W \xi \int d \mathbf{r} \frac{\partial\left(\nabla_{\alpha} \phi_{n}\right)}{\partial \mathbf{R}_{n}}\left(\nabla_{\beta} \phi_{n}\right) n_{\alpha}^{0} n_{\beta}^{0} \\
=\frac{K}{2 R_{c}^{2}} \int d \mathbf{r} \frac{\partial \phi_{n}}{\partial \mathbf{R}_{n}} \tanh \left[R_{c}^{2}\left(\left(\nabla \cdot \mathbf{n}^{0}\right)^{2}+\left(\nabla \times \mathbf{n}^{0}\right)^{2}\right)\right] \\
\quad+W \xi \int d \mathbf{r} \frac{\partial\left(\nabla_{\alpha} \phi_{n}\right)}{\partial \mathbf{R}_{n}}\left[\frac{\nabla_{\alpha} \phi_{n}}{\left|\nabla \phi_{n}\right|} \nabla_{\beta} \phi_{n} n_{\beta}^{0}+\left|\nabla \phi_{n}\right| n_{\alpha}^{0}\right],
\end{aligned}
$$

where Eqs.(14) and (15) are for the nematic and smectic- $\mathrm{C}^{*}$ solvents, respectively. These forms are very convenient because one can compute both $\partial \phi_{n} / \partial \mathbf{R}_{n}$ and $\partial\left(\nabla_{\alpha} \phi_{n}\right) / \partial \mathbf{R}_{n}$ at any time since $\phi_{n}$ is an analytical function of $\mathbf{R}_{n}$.

(iii) Finally, update the particle positions according to appropriate equations of motion such as

$$
m_{n} \frac{d^{2} \mathbf{R}_{n}}{d t^{2}}=\mathbf{f}_{n}^{P P}+\mathbf{f}_{n}^{P S}+\mathbf{f}_{n}^{H}+\mathbf{f}_{n}^{R},
$$

where $\mathbf{f}_{n}^{P P}$ is the force due to direct particle-particle interactions (hard or soft sphere for instance), $\mathbf{f}_{n}^{H}$ and $\mathbf{f}_{n}^{R}$ are the hydrodynamic and random forces. Repeating the steps (i) (iii) enables us to perform first-principles mesoscopic simulations for the dispersions containing many particles without neglecting many-body interactions.

\section{Results}

\subsection{Director configurations around a single particle}

We have performed simple demonstrations for two-dimensional (2D) LC colloid dispersions to test the performance of our procedure. The demo system has $100 \times 100$ lattice sites in a square box with a linear length $L=100$. Other physical parameters are chosen rather arbitrarily as $R_{c}=1, a=5$, and $\xi=2$, where the unit of length is the lattice spacing $l$. Since the director configurations in $2 \mathrm{D}$ can be expressed by a single scalar field $\theta(\mathbf{r})$, the tilt angle of the director against the horizontal $(x-)$ direction, Eq.(12) then reduces to

$$
\frac{\delta \mathcal{F}}{\delta \theta(\mathbf{r})}=\frac{\partial n_{\alpha}(\mathbf{r})}{\partial \theta(\mathbf{r})} \frac{\delta \mathcal{F}}{\delta n_{\alpha}(\mathbf{r})}=0 .
$$

The boundary condition is fixed at $\theta(\mathbf{r})=0$ at the edge of the box to avoid rotations of the reference frame. 

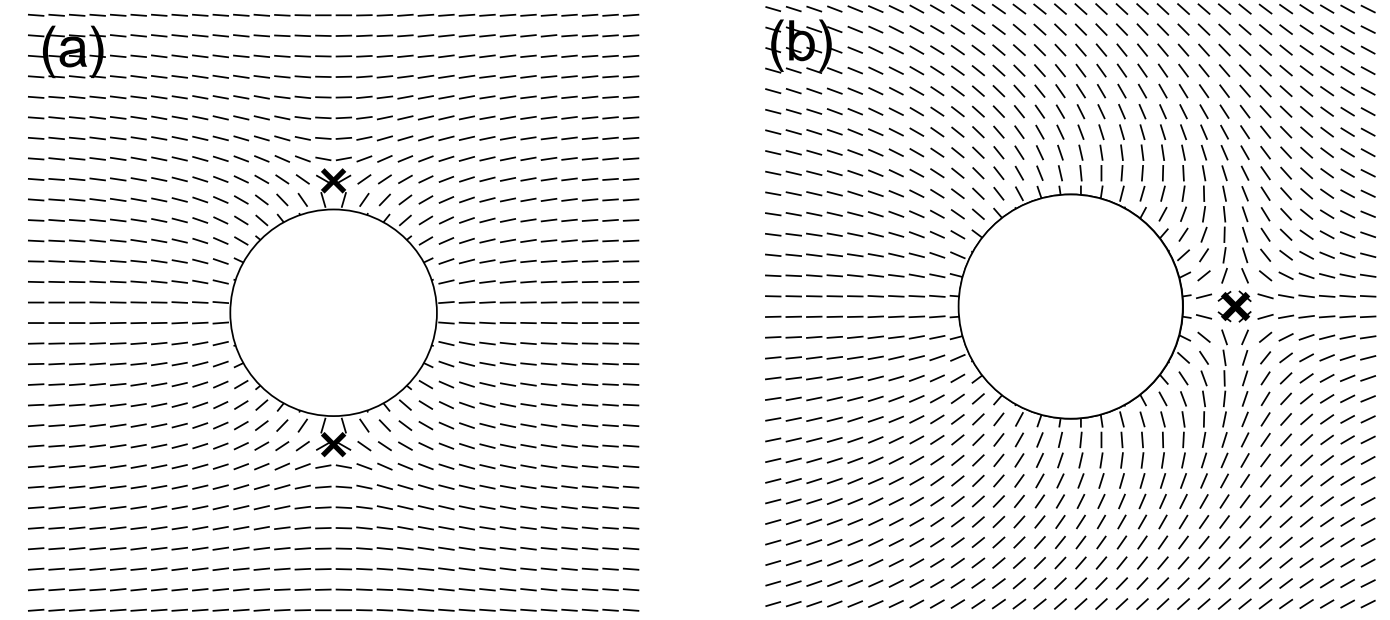

Figure 2. (a) Director configuration around a single particle immersed in nematic solvent with strong anchoring condition $W a / K=4$. The crosses show two $-1 / 2$ charge point defects and the obtained configuration has a quadrupolar character. (b) Director configuration around a single particle immersed in smectic- $\mathrm{C}^{*}$ solvent with $W a / K=5$. The cross shows -1 charge point defect and the obtained configuration has a dipolar character. The white disks indicate the colloid particles. Only $9 \%$ of the total system is shown for display purpose.

We first calculated the stable director configurations around a single particle immersed in 2D nematic and smectic- $\mathrm{C}^{*}$ solvents and showed them in Figure 2 (a) and (b), respectively. For the nematic solvent (a), the particle is accompanied by two $-1 / 2$ charge point defects with a strong anchoring condition $W a / K=4$. The distance between the defects and the particle center is about $1.2 a$, which is similar to the analytic value $1.236 a$ [34, and the director configuration around the single particle possess quadrupolar symmetries. This would correspond to the Saturn ring configuration observed in three dimensional (3D) systems. Although in principle particles can be accompanied by one -1 charge hedgehog defect in $2 \mathrm{D}$ as well as in $3 \mathrm{D}$, such configurations are unstable in the present $2 \mathrm{D}$ system since the elastic penalty of having $m$ point defects with charge $c$ scales as $m K c^{2}$. This was directly confirmed by recent simulations with perfect normal anchoring [34] and also by our simulations. For the smectic- $\mathrm{C}^{*}$ solvent (b) on the other hand, the particle is accompanied by one -1 charge point defect with an anchoring condition $W a / K=5$. The distance between the defect and the particle center is about $1.4 a$ which is again similar to the experimental value $1.4 a \pm 0.1 a[21$ and the analytic one $\sqrt{2} a[23$. Note that the director configuration around the particle possess dipolar symmetries similar to the experiment [21].

\section{2. aggregation of colloids in $L C$ solvents}

We have simulated the aggregation and ordering process of 30 and 10 colloid particles in nematic and smectic- $\mathrm{C}^{*}$ solvents, respectively, after the isotropic to nematic or isotropic to smectic- $\mathrm{C}^{*}$ transition occurred. Here we used the periodic boundary condition and 
set $W a / K=4$ (nematic) or 5 (smectic $\mathrm{C}^{*}$ ). Other parameters are the same as in the previous single particle case. The simulation was performed starting from a random particle configuration which is a typical configuration when the solvent is in the isotropic phase $(K=0)$. We then set $K=1$ and calculated $\mathbf{f}_{n}^{P S}$ according to the present procedure. The particle configurations were updated by numerically solving the steepest descent equation,

$$
\zeta \frac{d \mathbf{R}_{n}}{d t}=\mathbf{f}_{n}^{P S}+\mathbf{f}_{n}^{P P}
$$

which is obtained by simply substituting $d^{2} \mathbf{R}_{n} / d t^{2}=0, \mathbf{f}_{n}^{R}=0$, and $\mathbf{f}_{n}^{H}=-\zeta d \mathbf{R}_{n} / d t$ in Eq.(16). $\zeta=1$ is a friction constant and thus the off-diagonal components of the hydrodynamic interaction were not considered. Here we obtain

$$
\mathbf{f}_{n}^{P P}=-\frac{\partial E_{P P}}{\partial \mathbf{R}_{n}}
$$

from the repulsive part of the Lennard-Jones potential,

$$
E_{P P}=0.4 \sum_{n=1}^{N-1} \sum_{m=n+1}^{N}\left[\left(\frac{2 a}{\left|\mathbf{r}_{n}-\mathbf{r}_{m}\right|}\right)^{12}-\left(\frac{2 a}{\left|\mathbf{r}_{n}-\mathbf{r}_{m}\right|}\right)^{6}+\frac{1}{4}\right]
$$

truncated at the minimum distance $\left|\mathbf{r}_{n}-\mathbf{r}_{m}\right|=2^{7 / 6} a$, to avoid the particles overlapping each other within the colloid radius $\simeq a$. Snapshots from the present simulation are shown in Figures 3 and 4 for dispersions in nematic and smectic $C^{*}$ solvemts, respectively. In Figure 3, the particles are forming ordered clusters due to the quadrupolar attractive interaction among them. In Figure 4 on the other hand, the particles are forming string-like clusters due to the dipolar attractive interaction among them. Similar results have been obtained recently by experiments [21. Note that only up to two particle simulations have been done so far [8] and simulations of more than two particles would be extremely difficult or almost impossible even for $2 \mathrm{D}$ systems by means of other methods ever proposed.

\section{Concluding Remarks}

In summary, we have developed a powerful simulation method to investigate colloid dispersions interacting via solvents. We proposed a free energy functional which is suitable for MD type simulations. The following modifications have been made to the original Frank free energy functional.

(i) The coupling between the nematic solvent and particles at the interfaces is introduced explicitly through a smooth interface so that we can analytically calculate the force acting on each particle mediated by the host by taking derivatives of the free energy according to the particle positions.

(ii) The value of the free energy density is bounded semi-empirically to avoid a mathematical divergence in the defect centers. 

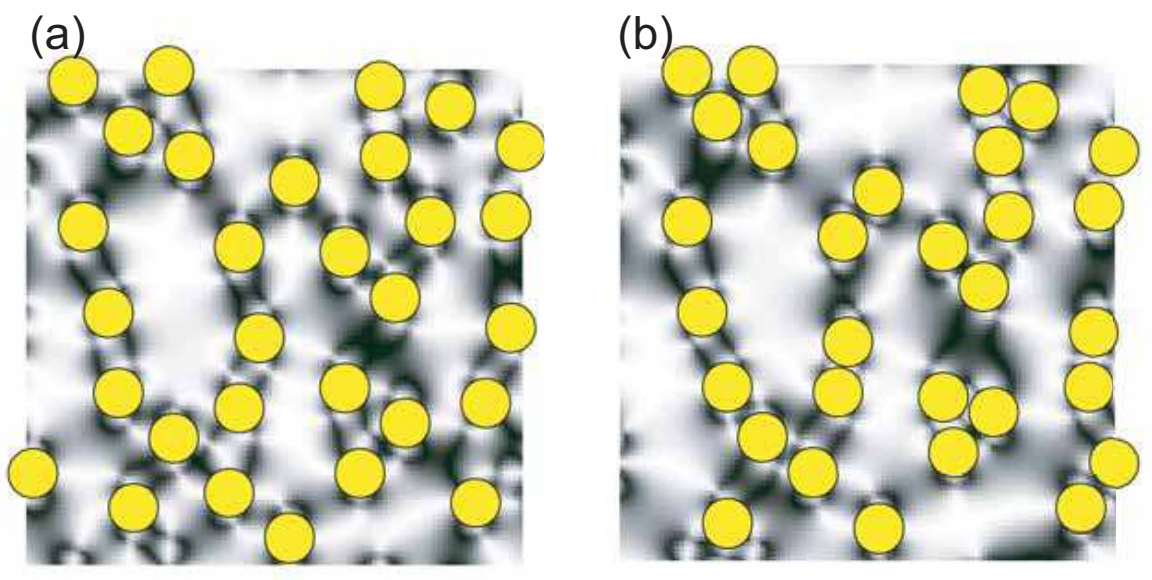

(c)

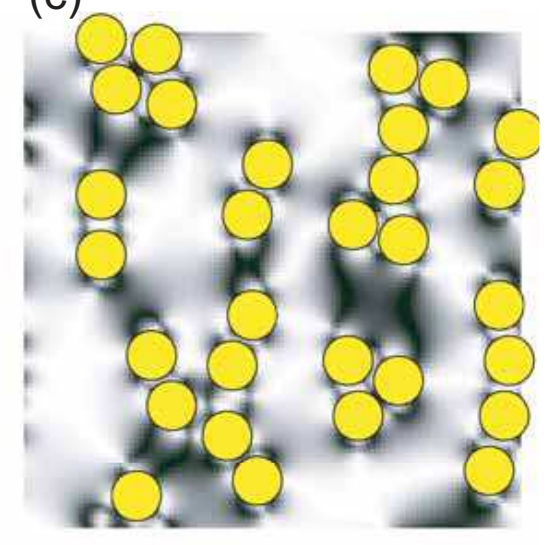

(d)

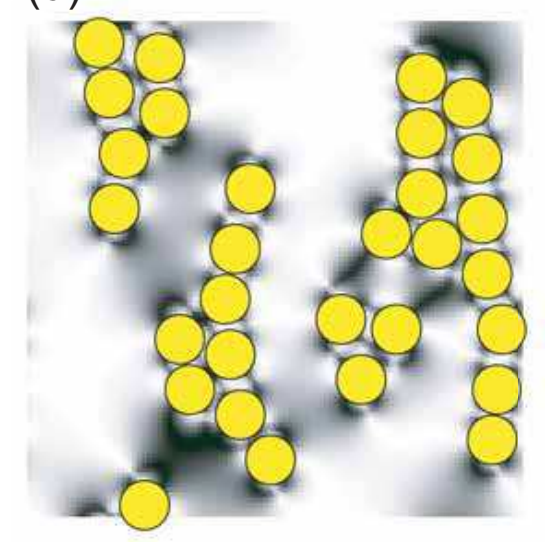

(e)

(f)
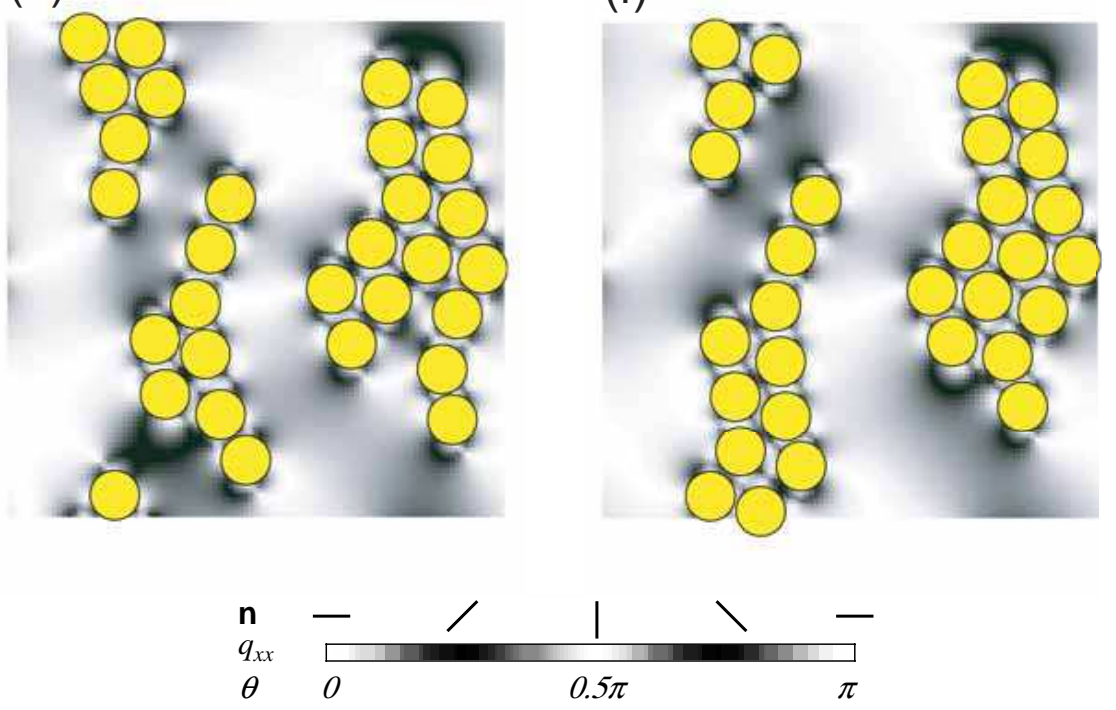

Figure 3. The aggregation and ordering process of colloid particles after the solvent exhibit the isotropic $(K=0)$ to nematic $(K=1)$ phase transition; (a) $t=0$, (b) $t=0.4$, (c) $t=1.6$, (d) $t=6.4$, (e) $t=16$, and (f) $t=40$. Each particle is accompanied by two $-1 / 2$ charge point defects. Darkness presents the value of $q_{x x}^{2}$. Black and white correspond to $q_{x x}^{2}=0$ and 0.25 , respectively. Those correspond also to $\theta=0.25 \pi, 0.75 \pi$ and $\theta=0,0.5 \pi, \pi$ as shown in the gradation map. 
(a)

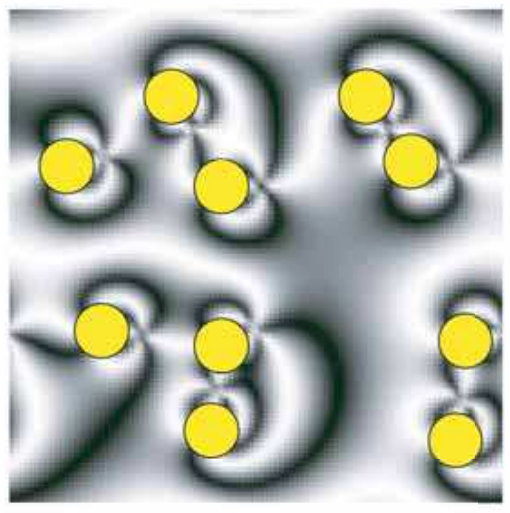

(c)

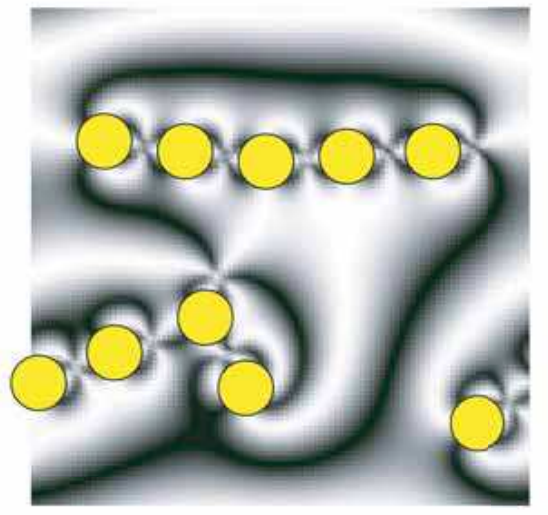

(e)

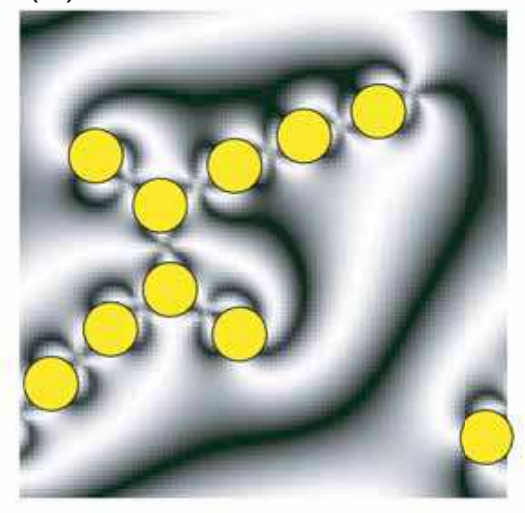

(b)

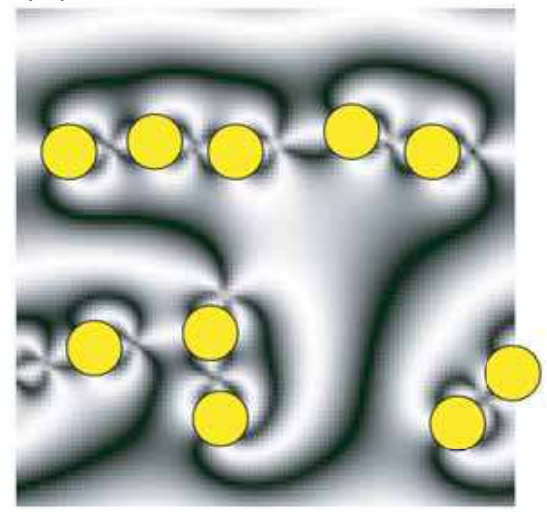

(d)

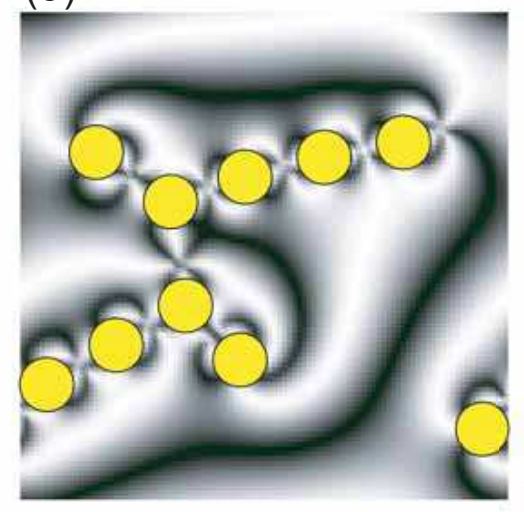

(f)

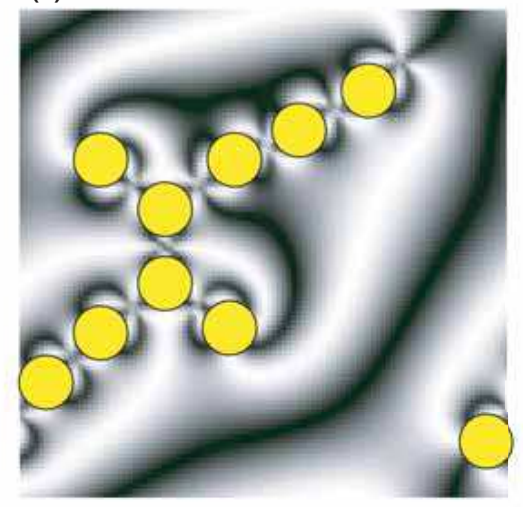

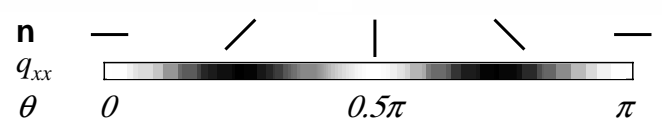

Figure 4. The aggregation and ordering process of colloid particles after the solvent exhibit the isotropic $(K=0)$ to smectic- $\mathrm{C}^{*}(K=1)$ phase transition; (a) $t=0$, (b) $t=0.25$, (c) $t=1$, (d) $t=4$, (e) $t=10$, (f) $t=25$. Each particle is accompanied by one -1 charge point defect. Darkness presents the value of $q_{x x}^{2}$. Black and white correspond to $q_{x x}^{2}=0$ and 0.25 , respectively. Those correspond also to $\theta=0.25 \pi, 0.75 \pi$ and $\theta=0,0.5 \pi, \pi$ as shown in the gradation map. 
We have performed demonstrations for 2D dispersions and confirmed that the method works quite well for systems which contain defects. Applications of this method to 3D systems should have no theoretical difficulties, but require somewhat heavier computation. This should allow the simulation of the chaining of the particles caused by the possible dipolar symmetry of the nematic configurations around a single particle. Although we have shown only simple demonstrations of the method by performing simulations of the 2D system in this letter, simulations with physically more interesting situations such as systems with non-circular particles, asymmetric particle pairs with different particle size, or particles with non-normal anchoring as well as more realistic simulations in 3D systems are possible. Efforts for taking into account the hydrodynamic effects are now underway by using smooth interface instead of imposing hydrodynamic boundary conditions at the colloid interface.

\section{Acknowledgements}

RY thanks Professor J.P. Hansen, Professor H. Löwen, and Dr. E. Terentjev for helpful discussions. A part of the present calculations have been carried out at the Human Genome Center, Institute of Medical Science, University of Tokyo.

\section{Appendix A. numerical implementation for $2 \mathrm{D}$ nematic}

Here, the superscripts $i, j(=1,2, \cdots, L)$ denote positions on a $2 \mathrm{D}$ lattice $(L \times L)$.

$$
\begin{aligned}
\mathcal{F}_{e l}= & \frac{K}{4 R_{c}^{2}} \sum_{i, j}\left(1-\sum_{n=1}^{N} \phi_{n}^{i, j}\right) \tanh \epsilon^{i, j} \\
\epsilon^{i, j}= & \frac{R_{c}^{2}}{2 l^{2}}\left[\left(q_{\alpha \beta}^{i, j}-q_{\alpha \beta}^{i-1, j}\right)^{2}+\left(q_{\alpha \beta}^{i+1, j}-q_{\alpha \beta}^{i, j}\right)^{2}\right. \\
& \left.+\left(q_{\alpha \beta}^{i, j}-q_{\alpha \beta}^{i, j-1}\right)^{2}+\left(q_{\alpha \beta}^{i, j+1}-q_{\alpha \beta}^{i, j}\right)^{2}\right] \\
q_{\alpha \beta}^{i, j}= & n_{\alpha}^{i, j} n_{\beta}^{i, j}-\delta_{\alpha \beta} / 2 \quad \frac{W \xi}{2} \sum_{i, j} \sum_{n=1}^{N}\left[\frac{1}{2}\left(\nabla_{\alpha} \phi_{n}^{i, j}\right)^{2}-\left(\nabla_{\alpha} \phi_{n}^{i, j}\right)\left(\nabla_{\beta} \phi_{n}^{i, j}\right) q_{\alpha \beta}^{i, j}\right] \\
\mathcal{F}_{s}= & \frac{\partial \mathcal{F}}{\partial \mathcal{F}} \frac{\partial q_{\alpha \beta}^{i, j}}{\partial q_{\alpha \beta}^{i, j}}=\frac{\partial \mathcal{F}}{\partial \theta^{i, j}} \frac{\partial q_{x x}^{i, j}}{\partial q_{x x}^{i, j}}+2 \frac{\partial \mathcal{F}}{\partial q_{x y}^{i, j}} \frac{\partial q_{x y}^{i, j}}{\partial \theta^{i, j}}+\frac{\partial \mathcal{F}}{\partial q_{y y}^{i, j}} \frac{\partial q_{y y}^{i, j}}{\partial \theta^{i, j}} \\
\frac{\partial \theta^{i, j}}{2} & =2 \sin \theta^{i, j} \cos \theta^{i, j}\left(\frac{\partial \mathcal{F}}{\partial q_{y y}^{i, j}}-\frac{\partial \mathcal{F}}{\partial q_{x x}^{i, j}}\right)+2\left(\cos ^{2} \theta^{i, j}-\sin ^{2} \theta^{i, j}\right) \frac{\partial \mathcal{F}}{\partial q_{x y}^{i, j}} \\
\frac{\partial \mathcal{F}}{\partial q_{\alpha \beta}^{i, j}}= & \frac{\partial \mathcal{F} \mathcal{F}_{e l}}{\partial q_{\alpha \beta}^{i, j}}+\frac{\partial \mathcal{F} \mathcal{F}_{s}}{\partial q_{\alpha \beta}^{i, j}}
\end{aligned}
$$




$$
\begin{aligned}
\frac{\partial \mathcal{F}_{e l}}{\partial q_{\alpha \beta}^{i, j}=} & \frac{K}{4 l^{2}}\left[\left(1-\sum_{n=1}^{N} \phi_{n}^{i, j}\right) \cosh ^{-2} \epsilon^{i, j}\left(4 q_{\alpha \beta}^{i, j}-q_{\alpha \beta}^{i-1, j}-q_{\alpha \beta}^{i+1, j}-q_{\alpha \beta}^{i, j-1}-q_{\alpha \beta}^{i, j+1}\right)\right. \\
& +\left(1-\sum_{n=1}^{N} \phi_{n}^{i-1, j}\right) \cosh ^{-2} \epsilon^{i-1, j}\left(q_{\alpha \beta}^{i, j}-q_{\alpha \beta}^{i-1, j}\right) \\
& +\left(1-\sum_{n=1}^{N} \phi_{n}^{i+1, j}\right) \cosh ^{-2} \epsilon^{i+1, j}\left(q_{\alpha \beta}^{i, j}-q_{\alpha \beta}^{i+1, j}\right) \\
& +\left(1-\sum_{n=1}^{N} \phi_{n}^{i, j-1}\right) \cosh ^{-2} \epsilon^{i, j-1}\left(q_{\alpha \beta}^{i, j}-q_{\alpha \beta}^{i, j-1}\right) \\
& \left.+\left(1-\sum_{n=1}^{N} \phi_{n}^{i, j+1}\right) \cosh ^{-2} \epsilon^{i, j+1}\left(q_{\alpha \beta}^{i, j}-q_{\alpha \beta}^{i, j+1}\right)\right] \\
\frac{\partial \mathcal{F}_{s}}{\partial q_{\alpha \beta}^{i, j}=} & -\frac{W \xi}{2} \sum_{n=1}^{N} \nabla_{\alpha} \phi_{n}^{i, j} \nabla_{\beta} \phi_{n}^{i, j} \\
\mathbf{f}_{n}^{P S}= & \frac{K}{4 R_{c}^{2}} \sum_{i, j} \frac{\partial \phi_{n}^{i, j}}{\partial \mathbf{R}_{n}} \tanh \epsilon^{(0) i, j}+W \xi \sum_{i, j} \frac{\partial\left(\nabla_{\alpha} \phi_{n}^{i, j}\right)}{\partial \mathbf{R}_{n}}\left(\nabla_{\beta} \phi_{n}^{i, j}\right) q_{\alpha \beta}^{(0) i, j} .
\end{aligned}
$$

\section{Appendix B. numerical implementation for $2 \mathrm{D}$ smectic-C*}

$$
\begin{aligned}
\mathcal{F}_{e l}= & \frac{K}{2 R_{c}^{2}} \sum_{i, j}\left(1-\sum_{n=1}^{N} \phi_{n}^{i, j}\right) \tanh \epsilon^{i, j} \\
\epsilon^{i, j}= & \frac{R_{c}^{2}}{2 l^{2}}\left[\left(n_{\alpha}^{i, j}-n_{\alpha}^{i-1, j}\right)^{2}+\left(n_{\alpha}^{i+1, j}-n_{\alpha}^{i, j}\right)^{2}\right. \\
& \left.+\left(n_{\alpha}^{i, j}-n_{\alpha}^{i, j-1}\right)^{2}+\left(n_{\alpha}^{i, j+1}-n_{\alpha}^{i, j}\right)^{2}\right] \\
\mathcal{F}_{s}= & W \xi \sum_{i, j} \sum_{n=1}^{N}\left[\left(\nabla \phi_{n}^{i, j}\right)^{2}-\left|\nabla \phi_{n}^{i, j}\right| \nabla \phi_{n}^{i, j} \cdot \mathbf{n}^{i, j}\right] \\
\frac{\partial \mathcal{F}}{\partial \theta^{i, j}}= & \frac{\partial \mathcal{F}}{\partial n_{\alpha}^{i, j}} \frac{\partial n_{\alpha}^{i, j}}{\partial \theta^{i, j}}=\frac{\partial \mathcal{F}}{\partial n_{x}^{i, j}} \frac{\partial n_{x}^{i, j}}{\partial \theta^{i, j}}+\frac{\partial \mathcal{F}}{\partial n_{y}^{i, j}} \frac{\partial n_{y}^{i, j}}{\partial \theta^{i, j}} \\
= & -n_{y}^{i, j}\left(\frac{\partial \mathcal{F}_{e l}}{\partial n_{x}^{i, j}}+\frac{\partial \mathcal{F}_{s}}{\partial n_{x}^{i, j}}\right)+n_{x}^{i, j}\left(\frac{\partial \mathcal{F}_{e l}}{\partial n_{y}^{i, j}}+\frac{\partial \mathcal{F}_{s}}{\partial n_{y}^{i, j}}\right) \\
\frac{\partial \mathcal{F}_{e l}}{\partial n_{\alpha}^{i, j}}= & \frac{K}{2 l^{2}}\left[\left(1-\sum_{n=1}^{N} \phi_{n}^{i, j}\right) \cosh ^{-2} \epsilon^{i, j}\left(4 n_{\alpha}^{i, j}-n_{\alpha}^{i-1, j}-n_{\alpha}^{i+1, j}-n_{\alpha}^{i, j-1}-n_{\alpha}^{i, j+1}\right)\right. \\
& +\left(1-\sum_{n=1}^{N} \phi_{n}^{i-1, j}\right) \cosh ^{-2} \epsilon^{i-1, j}\left(n_{\alpha}^{i, j}-n_{\alpha}^{i-1, j}\right)
\end{aligned}
$$




$$
\begin{gathered}
+\left(1-\sum_{n=1}^{N} \phi_{n}^{i+1, j}\right) \cosh ^{-2} \epsilon^{i+1, j}\left(n_{\alpha}^{i, j}-n_{\alpha}^{i+1, j}\right) \\
+\left(1-\sum_{n=1}^{N} \phi_{n}^{i, j-1}\right) \cosh ^{-2} \epsilon^{i, j-1}\left(n_{\alpha}^{i, j}-n_{\alpha}^{i, j-1}\right) \\
\left.+\left(1-\sum_{n=1}^{N} \phi_{n}^{i, j+1}\right) \cosh ^{-2} \epsilon^{i, j+1}\left(n_{\alpha}^{i, j}-n_{\alpha}^{i, j+1}\right)\right] \\
\frac{\partial \mathcal{F}_{s}}{\partial n_{\alpha}^{i, j}=}-W \xi \sum_{n=1}^{N}\left|\nabla \phi_{n}^{i, j}\right| \nabla_{\alpha} \phi_{n}^{i, j} \\
\mathbf{f}_{n}^{P S}=\frac{K}{2 R_{c}^{2}} \sum_{i, j} \frac{\partial \phi_{n}^{i, j}}{\partial \mathbf{R}_{n}} \tanh \epsilon^{(0) i, j} \\
+W \xi \sum_{i, j} \frac{\partial\left(\nabla_{\alpha} \phi_{n}^{i, j}\right)}{\partial \mathbf{R}_{n}}\left(\left|\nabla \phi_{n}^{i, j}\right| n_{\alpha}^{i, j}+\frac{\nabla \phi_{n}^{i, j} \cdot \mathbf{n}^{i, j}}{\left|\nabla \phi_{n}^{i, j}\right|} \nabla_{\alpha} \phi_{n}^{i, j}\right)
\end{gathered}
$$

\section{References}

[1] W.B. Russel, D.A. Saville, and W.R. Schowalter, Colloidal Dispersions (Cambridge University Press, Cambridge, 1995).

[2] V.M. Mostepanenko and N.N. Trunov, The Casimir Effect and its Application (Clarendon Press, Oxford, 1997).

[3] A. Borštnik, H, Stark, and S. Žumer, Phys. Rev. E 60, 4210 (1999).

[4] H. Stark, Phys. Rep. 351, 387 (2001).

[5] E.M. Terentjev, Phys. Rev. E 51, 1330 (1995).

[6] S. Ramaswamy, R. Nityananda, V.A. Raghunathan, and J. Prost, Mol. Crys. Liq. Crys. 288, 175 (1996).

[7] R.W. Ruhwandl and E.M. Terentjev, Phys. Rev. E 56, 5561 (1997).

[8] H. Stark, Eur. Phys. J. B 10, 311 (1999); H. Stark, J. Stelzer, and R. Bernhard, Eur. Phys. J. B 10, 515 (1999).

[9] O. Mondain-Monval, J.C. Dedieu, T. Gulik-Krzywicki, and P. Poulin, Eur. Phys. J. B 12, 567 (1999).

[10] Y.G. Gu and N.L. Abbott, Phys. Rev. Lett. 85, 4719 (2000).

[11] P. Poulin, H. Stark, T.C. Lubensky, and D.A. Weitz, Science 275, 1770 (1997); P. Poulin, V. Cabuil, and D.A. Weitz, Phys. Rev. Lett. 79, 4862 (1997); P. Poulin and D.A. Weitz, Phys. Rev. E 57, 626 (1998).

[12] R.W. Ruhwandl and E.M. Terentjev, Phys. Rev. E 55, 2958 (1997).

[13] T.C. Lubensky, D. Pettey, N. Currier, and H. Stark, Phys. Rev. E 57, 610 (1998).

[14] B.I. Lev and P.M.Tomchuk, Phys. Rev. E 59, 591 (1999).

[15] V.A. Raghunathan, P. Richetti, and D. Roux, Langmuir 12, 3789 (1996); V.A. Raghunathan, P. Richetti, D. Roux, F. Nallet, and K. Sood, Langmuir 16, 4720 (2000).

[16] S.P. Meeker, W.C.K. Poon, J. Crain, and E.M. Terentjev, Phys. Rev. E 61, R6083 (2000); V.J. Anderson, E.M. Terentjev, S.P. Meeker, J. Crain, and W.C.K. Poon, Eur. Phys. J. E 1, 11 (2001); V.J. Anderson, E.M. Terentjev, Eur. Phys. J. E 1, 21 (2001).

[17] J.C. Loudet, P. Barios, and P. Poulin, Nature 407, 611 (2000).

[18] J. Yamamoto and H. Tanaka, Nature 409, 321 (2001).

[19] V.G. Nazarenko, A.B. Nych, and B.I. Lev, Phys. Rev. Lett. 87, 075504 (2001).

[20] T. Yamamoto, J. Yamamoto, B.I. Lev, and H. Yokoyama, Appl. Phys. Lett. 81, 2187 (2002). 
[21] P. Cluzeau, P. Poulin, G. Joly, and H.T. Nguyen, Phys. Rev. E 63, 031702 (2001).

[22] P. Patricio, M. Tashinkevych, and M.M. Telo da Gama, Eur. Phys. J. E 7, 117 (2002).

[23] D. Petty, T.C. Lubensky, and D.R. Link, Liquid Crystals 25, 579 (1998).

[24] C.M. Care, L. Halliday, K. Good, and S.V. Lishchuk, Phys. Rev. E 67, 061703 (2003).

[25] J. Fukuda, the article in this issue.

[26] Y. Nakayama and R. Yamamoto, unpublished.

[27] K. Kim and R. Yamamoto, unpublished.

[28] J.L. Billeter and R.A. Pelcovits, Phys. Rev. E 62, 711 (2000).

[29] D. Andrienko, G. Germano, and M.P. Allen, Phys. Rev. E 63, 041701 (2001).

[30] H. Löwen, P.A. Madden, and J.P Hansen, Phys. Rev. Lett. 68, 1081 (1992); H. Löwen, J.P Hansen, and P.A. Madden, J. Chem. Phys. 98, 3275 (1993).

[31] P.G. de Gennes and J. Prost, The Physics of Liquid Crystals, 2nd ed. (Clarendon, Oxford, 1993).

[32] R. Yamamoto, Phys. Rev. Lett. 87, 075502 (2001).

[33] H. Tanaka and T. Araki, Phys. Rev. Lett. 85, 1338 (2000).

[34] J. Fukuda and H. Yokoyama, Eur. Phys. J. E 4, 389 (2001); J. Fukuda, M. Yoneya, and H. Yokoyama, Phys. Rev. E 65, 041709 (2002). 